\title{
THE MOLECULAR THEORY OF POLARIZED EMISSION: LINEAR, CIRCULAR, AND MAGNETICALLY INDUCED CIRCULAR POLARIZATION OF EMISSION
}

\author{
K.W. HIPPS \\ Department of Chemistry, The University of Michigan, Ann Arbor, Michigan 48109, USA
}

Received 17 November 1976

Revised manuscript received 21 March 1977

\begin{abstract}
Quantum field theory is used to derive transition probabilities pertinent to natural and magnetically induced polarized emission. First order terms in magnetic field strength are considered, while second order terms are neglected. It is shown that the existing absorption theory may be used for the emission case provided that (a) a frequency dependent factor is used, (b) level population terms are handled in the proper manner, and (c) superposition of the contributions from the various emitting levels is performed in such a way as to take account of the radiationless transition probabilities. Special emphasis is given to the parameterization of the magnetically induced circular polarization of emission.
\end{abstract}

\section{Introduction}

There is a growing interest in the phenomena of polarized luminescence. In the last few years there have appeared a number of experimental studies of the natural [e.g. 1] and magnetically induced [e.g. 2] circular polarization of emission. Snir and Schellman [3] and Steinberg and Ehrenberg [4] have presented theoretical studies of the role of brownian motion and photoselection in certain cases of natural circular polarization of emission (CPE). More recently, Riehl and Richardson [5] have presented the first general theory of CPE and its magnetically induced analog (MICE) based on the formalism of Powers and Thirunamachandran [6] which employs quantum electro-dynamics. Their paper includes discussion of both photoselection and rotatory brownian motion and the results are presented in a format similar to that of Eyring and Caldwell [7] for the absorption case.

This work represents an independent development [2a] of the theory of polarized emission. The present formalism being closely related to that of Stephens $[8,9]$ but employing quantum electro-dynamics for the calculation of transition probabilities. In addition to the formalistic differences between this work and that of Riehl and Richardson, there are several substantive differences. This work emphasises the role of the molecular Hamiltonian in determining the form of the interaction Hamiltonian. Several interaction terms appear in this paper which are absent in theirs. A discussion of their magnitude and when they may be neglected is presented. Further, the present paper treats the effects on the observed spectrum when several electronic states contribute to the observed emission. As a consequence, special attention is given to the role of radiationless decay processes. The Riehl and Richardson paper, on the other hand, presents detailed calculations of the effects of photoselection and rotatory brownian motion on the observed CPE and MICE spectra; the present work restricts its attention to emission observed from a sample of fixed, but arbitrary, orientational distribution. Both the present paper and that of Riehl and Richardson assume unpolatized radiation as the excitation source.

The end result of section 3 is the calculation of the probability of emission of a photon of arbitrary polarization from a molecule having a Hamiltonian of rather general form. This transition probability equation is written in terms of molecule fixed coordinates, and as such, represents the starting point for all calculations involving orientation and distribution averaging. The later sections confine themselves to particularizing this general result to the parameterization and identification of terms in the formalism of Stephens $[8,9]$. The CPE and MICE pa- 
rameterization is carried out only for the $180^{\circ}$ "head on" experimental geometry. Riehl and Richardson present results for the $90^{\circ}$ geometry as well in their formulation. MICE calculations are presented to first order in applied magnetic field strength.

Our general theoretical approach in the first sections of this paper will now be outlined. The molecular Hamiltonian, in the presence of the radiation field, will be constructed from the field free Hamiltonian. This Hamiltonian will then be separated into three terms; the field free molecular Hamiltonian, the Zeeman interaction Hamiltonian, and the radiation interaction Hamiltonian. Finally, we will append to this the Hamiltonian for the radiation field in charge free space. The eigenstates of the field free molecular and charge free radiation Hamiltonians will be used as a basis set for the system states. The system states will be constructed via first order perturbation theory. The quantization of the radiation field is the province of quantum electrodynamics. At its present state of development, it is textbook material [10-12] and the reader is assumed to have some familiarity with the basic formalism. A much more detailed account of the quantization problem as it relates to the present work is available in ref. [2a]:

\section{Hamiltonians}

We first consider the problem of the molecular Hamiltonian in the presence of both a static magnetic field and the radiation field, $\underline{H}^{\mathrm{MF}}$. This will be decomposed into terms involving the field free molecular Hamiltonian, $\underline{H}^{0}$, the first order static magnetic field term, $\underline{H}^{\mathrm{Z}}$, and the radiation interaction term, $\underline{H}^{\mathrm{I}}$.

$$
\underline{H}^{\mathrm{MF}}=\underline{H}^{\mathrm{M}}+\underline{H}^{\mathrm{I}}=\underline{H}^{0}+\underline{H}^{\mathrm{Z}}+\underline{H}^{\mathrm{I}} \text {. }
$$

In order that our formalism be appi: able to inorganic complexes incorporating metals of the transition and rare earth series, $\underline{H}^{0}$ is chosen to incluac electronic spin-orbit coupling and electronic spin-spin coupling. Further, we do not limit the electronic coupling to a given center but allow for the full molecular contribution. Without further discussion, we adopt the Hamiltonian used by Stephens in his treatment of MCD [9].

$$
\underline{H}^{0}=\sum_{i} \frac{p_{i}^{2}}{2 m_{i}}+\frac{1}{2 m^{2} c^{2}} \sum_{i} s_{i} \cdot \nabla_{i} V \times p_{i}+V+\sum_{i<j} \frac{e^{2}}{m^{2} c^{2}}\left[\left(s_{i} \cdot s_{j} / r_{i j}^{3}\right)-3\left(s_{i} \cdot r_{i j}\right)\left(s_{j} \cdot r_{i j}\right) / r_{i j}^{5}\right] \text {. }
$$

The molecular potential, $V$, is taken to be the Coulomb interaction between all nuclei and electrons. The spin dependent terms are to be summed over electronic coordinates only. It is important to notice that the eigenfunctions of $\underline{H}^{0}$ are dependent on both nuclear and electronic coordinates.

As is shown in many textbooks $[12,13]$, in the presence of an external field the canonical momentum is no longer the quantum analog of $m \dot{r}$. Instead, the momentum conjugate to $r_{i}$ is given by

$$
p_{i}=m v+e_{i} A_{i} / c \text {. }
$$

In order that eq. (2) be correct in the presence of an external field, we must replace $p_{i}$ by $p_{i}-e_{i} \underline{A}_{i} / c$, where $\underline{A}$ is the total vector potential due to all external fields. Further, the inclusion of spin in (2) requires that the spin-field interaction term, $(e / m c) \Sigma_{i} s_{i} \cdot \nabla \times \underline{A}_{i}$, also be included. These modifications convert $\underline{H}^{0}$ in to $\underline{H}^{\mathrm{MF}} \neq$.

$$
\begin{aligned}
& \underline{H}^{\mathrm{MF}}=\sum_{i} \frac{\left(p_{i}-e_{i} A_{i} / c\right)^{2}}{2 m_{i}}+\frac{1}{2 m^{2} c^{2}} \sum_{i} s_{i} \cdot \nabla_{i} V \times\left(p_{i}-e_{i} \underline{A}_{i} / c\right) \\
& \quad+V+\frac{e}{m c} \sum_{i} s_{i} \cdot \nabla \times \underline{A}_{i}+\sum_{i<j} \frac{e^{2}}{m^{2} c^{2}}\left[\left(s_{i} \cdot s_{j} / r_{i j}^{3}\right)-3\left(s_{i} \cdot r_{i j}\right)\left(s_{j} \cdot r_{i j}\right) / r_{i j}^{5}\right] .
\end{aligned}
$$

In order to perform the decomposition suggested in eq. (1), we must first identify the radiation and static field contributions to the total vector potential $\boldsymbol{A}_{i}$. This decomposition is given by

$\neq c$ is taken as positive. 


$$
A_{i}=A_{i}+A_{i}
$$

where the vector potential due to the static external magnetic field, $H$, is

$$
\mathcal{R}_{i}=\frac{1}{2} \boldsymbol{H} \times \boldsymbol{r}_{i}
$$

Substitution of (5) and (6) into eq. (4) yields, after much tedious algebra, the desired decomposition. The results are

$$
\underline{H}^{\mathrm{Z}} \equiv-\boldsymbol{\mu} \cdot \boldsymbol{H}
$$

where

$$
\left.-\mu^{\prime}=\sum \frac{e}{2 m c}\left(l_{i}+2 s_{i}\right)+\sum \frac{e}{4 m^{2} c^{3}}\left[\nabla_{i} V \cdot r_{i}\right) s_{i}-\left(s_{i} \cdot r_{i}\right) \nabla_{i} V\right]
$$

and

$$
\underline{H}^{\mathrm{I}}=-\sum A_{i} \cdot u_{i}+\sum \frac{e}{m c} \nabla \times A_{i} \cdot s_{i}
$$

where

$$
u_{i}=-\sum \frac{e}{m c}\left(p_{i}+\frac{1}{2 m c^{2}} s_{i} \times \nabla_{i} V+e A_{i} / c\right) .
$$

Because the electronic/nuclear mass ratio is small, terms in nuclear coordinates in eqs. (8) and (10) have been neglected. $\underline{H}^{\mathrm{I}}$ and $\underline{H}^{\mathrm{Z}}$ are now purely electronic operators. Further, we have made the approximation that terms in $A_{i} \cdot A_{i}$ and $A_{i} \cdot A_{i}$ are negligible. As long as we ignore zero and two photon process, we are forced into neglecting the term in $\boldsymbol{A}_{i} \cdot \boldsymbol{A}_{i}$. It is also important to note that within the approximation that $\boldsymbol{A}_{i} \cdot \boldsymbol{A}_{i}$ and $\boldsymbol{A}_{i} \cdot \boldsymbol{A}_{i}$ are zero, $A_{i} \cdot A_{i}$ will also be zero.

Obviously, we cannot provide the exact eigenfunctions of $\underline{H}^{\mathrm{M}}$; we can represent them formally as in eq. (11). The eigenfunctions of $\underline{H}^{0}$ may also be formally represented as in eq. (12).

$$
\underline{H}^{\mathrm{M}}|K\rangle=W_{K}|K\rangle, \quad \underline{H}^{0}|k\rangle=W_{k}|k\rangle,
$$

To complete our system Hamiltonian, we express the charge free radiation field Hamiltonian in terms of creation and annihilation operators for each mode, $k$, and polarization, $\lambda$. The dagger indicates the creation operator.

$$
\underline{H}^{F}=\sum_{k, \lambda}\left(a_{k \lambda}^{\dagger} a_{k \lambda}+\frac{1}{2}\right) \hbar k c
$$

Its eigenstates are given by (14) with energies demonstrated by (15).

$$
\left|\left\{n_{k \lambda}\right\}\right\rangle=\prod_{k, \lambda}\left|n_{k \lambda}\right\rangle, \quad H^{F}\left|\left\{n_{k \lambda}\right\}\right\rangle=\sum \hbar k c\left(n_{k \lambda}+\frac{1}{2}\right)\left|\left\{n_{k, \lambda}\right\}\right\rangle .
$$

Any operators or properties of the radiation field are now to be expressed in terms of these creation and annihilation operators. Specifically, the vector potential of the radiation field is given by

$$
A=\sum_{k, \lambda}(h c / k V)^{1 / 2}\left(\Pi_{k \lambda} \exp (-\mathrm{i} k \cdot r) a_{k \lambda}^{\dagger}+\Pi_{k \lambda}^{*} \exp (+\mathrm{i} k \cdot r) a_{k \lambda}\right),
$$

where the II's are the normalized polarization vectors and the star denotes the complex conjugate. We now use eq. (16) to expand $\underline{H}^{I}$ in terms of field and molecular operators. Since our interest is the emission case, we retain only those terms containing creation operators for the radiation field 


$$
\underline{H}^{i}=-\sum_{j} \sum_{k \cdot \lambda}(h c / k V)^{1 / 2}\left\{\exp \left(-\mathrm{i} k \cdot r_{j}\right)\left[\left(\Pi_{k \lambda} \cdot u_{j}\right)-\mathrm{i}(e / m c)\left(k \times s_{j} \cdot \boldsymbol{\Pi}_{k \lambda}\right)\right]\right\} a_{k \lambda}^{t}
$$

\section{Integrated transition probability}

From the previous sections we have available a Hamiltonian, $\underline{H}$, that represents the energy of the molecula plus radiation and static field

$$
\underline{H}=\underline{H}^{\mathrm{M}}+\underline{H}^{\mathrm{F}}+\underline{H}^{\mathrm{I}}
$$

Also at our disposal are the eigenstates of $\underline{H}^{\mathrm{F}}$ and $\underline{H}^{\mathrm{M}}$; these are factors of the direct product space that is a complete space for representation of $\underline{H}$. We define this space by

$$
\left.\|\left\{n_{k \lambda}\right\}\right\rangle=|1\rangle\left|\left\{n_{k \lambda}\right\}\right\rangle,
$$

where $n_{k \lambda}$ is the collection of quantum numbers, $n$, for all modes and polarizations. $[\mathrm{I}\rangle$ is an eigenstate of $\underline{H}^{\mathrm{M}}$. In general,

$$
\underline{H}] \phi\rangle=i \hbar \partial|\phi\rangle / \partial t, \quad|\phi\rangle=\sum_{F,\left\{n_{k \lambda}\right\}} C_{F,\left\{n_{k \lambda}\right\}}^{\phi}\left|\mathrm{F}\left\{n_{k \lambda}\right\}\right\rangle .
$$

With the tacit assumption that the system is prepared in the state $|1\{0\}\rangle$ before emission, direct application of first order perturbation theory allows us to calculate

$$
\left|C_{\mathrm{F}\left\{n_{k \lambda}\right\}}(t)\right|^{2}=t\left(8 \pi^{3} / h^{2}\right)\left|\left\{\mathrm{F}\left\{n_{k \lambda}\right\}\left|H^{\mathrm{I}}\right| \mathrm{I}\{0\}\right)\right|^{2} \delta(\Delta \omega) \text {. }
$$

Rather than becoming involved in the complicated, and generally unsolved, problem of line shapes for molecules, it is customary to consider the integrated transition probability per unit time interval. We define it by

$$
P\left[\mathrm{I}\{0\} \rightarrow \mathrm{F}\left\{n_{k \lambda}\right\}\right] \equiv \int\left|C_{\mathrm{F}\left\{n_{k \lambda}\right\}}(t)\right|^{2} / t \mathrm{~d} \omega .
$$

Therefore, using eq. (22)

$$
P\left[\mathrm{I}\{0\} \rightarrow \mathrm{F}\left\{n_{k \lambda}\right\}\right]=\left(8 \pi^{3} / h^{2}\right)\left|\left\langle\mathrm{F}\left\{n_{k \lambda}\right\}\left|\underline{H}^{\mathrm{l}}\right| \mathrm{I}\{0\}\right\rangle\right|^{2} .
$$

With the aid of eqs. (23) and (17) we have

where

$$
P\left[I\{0\} \rightarrow F\left\{\ldots 1_{k \lambda} \ldots\right\}\right]=\left(8 \pi^{3} c / h k V\right)\left|\left(F\left|\sum_{j} \exp \left(-\mathrm{i} k \cdot r_{j}\right)\left[u_{j}-\mathrm{i}(e / m c) k \times s_{j}\right]\right| \mathrm{I}\right\rangle \cdot \mathbf{\Pi}_{k \lambda}\right|^{2},
$$

$$
k=\omega_{\mathrm{IF}} / c .
$$

Because there is a continuum of possible $k$, we are experimentally limited to measuring the number of photons emitted into the solid angle $d \Omega_{k}$. Further, eq (25) must be interpreted as holding the length of $k$ within a variance $\mathrm{d} k=\mathrm{d} \omega$ ic about $\omega=\omega_{\mathrm{IF}}$. The measurable quantity is then the integrated probability of photon emission into the solid angle $d \Omega$. This is given by eq. (26) where $g\left(\omega_{\mathrm{IF}}\right)$ is the density of photon states in $k$ in the volume subtended by $d \Omega_{k}$ such that all those states on the interval $\left[\omega_{\mathrm{IF}}, \omega_{\mathrm{IF}}+\mathrm{d} \omega\right]$ are counted. The probability of emission in the solid angle $\mathrm{d} \Omega_{k}$ per unit time per unit frequency is therefore

$$
P^{k \lambda}[\mathrm{I} \rightarrow \mathrm{F}]=\left(\omega_{\mathrm{IF}} / h c\right)\left|M_{\mathrm{IF}}^{k \lambda}\right|^{2} P^{k \lambda}\left[\mathrm{I}\{0\} \rightarrow \mathrm{F}\left\{\ldots 1_{k \lambda} \ldots\right\}\right] g\left(\omega_{\mathrm{IF}}\right)
$$

where 


$$
g(\omega)=V k^{2} / 8 \pi^{3} c, \quad \omega_{\mathrm{IF}}=\left(E_{\mathrm{I}}-E_{\mathrm{F}}\right) / \hbar
$$

and

$$
M_{\mathrm{IF}}^{k}=\left\langle\mathrm{F}\left|\sum_{j} \exp \left(-\mathrm{i} k \cdot r_{j}\right)\left[u_{j}-\mathrm{i}(e / m c) k \times s_{j}\right]\right| \mathrm{I}\right\rangle
$$

or

$$
M_{\mathrm{IF}}^{k \lambda}=M_{\mathrm{IF}}^{k} \cdot \mathbf{H}_{k \lambda}
$$

\section{Dipole and quadrupole radiation}

It is customary to simplify the calculation of $M_{\mathbb{1 F}}^{k \lambda}$ by expanding the exponential in a Taylor series as

$$
\exp \left(-\mathrm{i} k \cdot r_{i}\right)=1-\mathrm{i} k \cdot r_{i}-\left(k \cdot r_{i}\right)^{2} / 2+\ldots .
$$

Because $k \cdot r_{i} \sim 3 \times 10^{-3}$ for visible wavelengths, we may very accurately neglect all but the first two terms. With this approximation

$$
M_{\mathrm{IF}}^{k \lambda}=\left\langle\mathrm{F}\left|\sum_{j} u_{j}\right| \mathrm{I}\right\rangle-\mathrm{i}\left(\mathrm{F}\left|\sum_{j}\left[\left(k \cdot r_{j}\right) u_{j}+(e / m c) k \times s_{j}\right]\right| \mathrm{I}\right\rangle
$$

We will now transform eq. (28) to the usual dipole and quadrupole terms. With the use of the commutator

$$
(2 \pi i / h c)\left[\underline{H}^{\mathrm{M}}, m\right]=u \quad \text { and } \quad m=\sum_{i} e_{i}
$$

we can convert the first term of eq. (28) to the'electric dipole term as

$$
M_{\mathrm{IF}}^{k \lambda}=-\mathrm{i}\left(\omega_{\mathrm{IF}} / c\right)\langle\mathrm{F}|m| \mathrm{I}\rangle-\mathrm{i}\left(\mathrm{F}\left|\sum_{j}\left\{\left(k \cdot r_{j}\right) u_{j}+(e / m c) k \times s_{j}\right\}\right| \mathrm{I}\right\rangle
$$

The term in $k \cdot r$ is expanded as

$$
\sum_{i}\left(k \cdot r_{j}\right) u_{j}=\frac{1}{2} \sum_{j}\left(k \cdot r_{j}\right) u_{j}+\left(k \cdot u_{j}\right) r_{j}+\frac{1}{2} \sum_{j}\left[\left(k \cdot r_{j}\right) u_{j}-\left(k \cdot u_{j}\right) r_{j}\right]
$$

The first term is usually called the quadrupole term and gives

$$
M_{\mathrm{IF}}^{k}=-\mathrm{i}\left(\omega_{\mathrm{IF}} / c\right)\langle\mathrm{F}|m| \mathrm{I})-\left(\omega_{\mathrm{IF}} / 2 c\right) k \cdot(\mathrm{F}|q| \mathrm{I}\rangle-\frac{\mathrm{i}}{2}\left\langle\mathrm{~F}\left|\sum_{j}\left[\left(k \cdot r_{j}\right) u_{j}-\left(k \cdot u_{j}\right) r_{j}+2(e / m c) k \times s_{j}\right]\right| \mathrm{D},\right.
$$

where

$$
q_{\alpha \beta}=q_{\alpha \beta}=\sum_{j} e r_{\alpha j} r_{\beta j}
$$

We will call $q$ the quadrupole tensor.

The last term in eq. (30) is called the magnetic dipole term. In order to demonstrate that it is requires more manipulation to give:

$$
-\frac{i}{2}(F|(k \cdot r) u-(k \cdot u) r+2(e / m c) k \times s| I\rangle=+i k \times\left\langle F\left|\mu^{\prime}+\xi\right| I\right\rangle
$$

where

$$
\xi=-\left(e^{2} / 4 m c^{2}\right) \sum_{j}\left[r_{j}^{2} H-\left(r_{j} \cdot H\right) r_{j}\right]
$$




\section{K.W. Hipps/Theory of polarized emission}

Collecting our results, we have

$$
M_{\mathrm{IF}}^{k \lambda}=-\mathrm{i}\left(\omega_{\mathrm{IF}} / c\right)\langle\mathrm{F}|m| \mathrm{I}\rangle+\mathrm{i} k \times\left\langle\mathrm{F}\left|\boldsymbol{\mu}^{\prime}+\xi\right| \mathrm{I}\right\rangle-\left(\omega_{\mathrm{IF}} / 2 c\right) k \cdot\langle\mathrm{F}|q| \mathrm{I}\rangle
$$

The second term in eq. (32) merits some discussion. I will call it the generalized magnetic dipole term because it becomes the usual term, $\mu$, when the external field and the spin-orbit coupling vanish. We can estimate the size if the $\xi$ term with respect to the $\mu$ term by taking $\mid\langle F|r| D| \approx 2$ atomic units $\left(2 a_{0}\right)$ and $|[F|\mu| I\rangle| \approx e h /(2 m c)$.

$\mid\left[F|\xi| I|X| \mid\langle F|\mu| D| \approx[2 e /(\bar{h} c)] a_{0}^{2} H \approx 10^{-9} H\right.$, where $H$ is in gauss. If $\langle F|\mu| \mathrm{I}\rangle$ does not vanish, even for a $10^{5}$ gauss field, $\langle F|\xi| I\rangle$ is still quite negligible. The spin-orbit term may be similarly evaluated

$$
\left|\left(\mathrm{F}\left|\boldsymbol{\mu}^{\prime}-\mu\right| \mathrm{I}\right\rangle\right| /|\langle\mathrm{F}|\boldsymbol{\mu}| \mathrm{I}\rangle| \approx \mid\left(\mathrm{F}|\nabla V \cdot \boldsymbol{r}| /\left(2 m c^{2}\right)\right. \text {. }
$$

Using a central field approximation, $\nabla V \cdot r \approx 2 m^{2} c^{2} \zeta(r)$, where $\xi(r)$ is the radial spin-orbit operator,

$\mid\left(\mathrm{F}|\nabla V \cdot r| \mathrm{I}\left|/\left(2 m c^{2}\right) \approx\right|\left(\mathrm{F}|\zeta| \mathrm{D} \mid m a_{0}^{2}\right.\right.$.

Using $\mid\left[F|\zeta| D||^{2} \sim|\langle|\zeta| I\rangle| \bar{h}^{2} \lesssim 10^{3} \mathrm{~cm}^{-1}\right.$ (the spin-orbit coupling constant), we have

$$
\left\langle\mathbf{F}\left|\boldsymbol{\mu}^{\prime}-\boldsymbol{\mu}\right| \mathrm{I}\right\rangle|/|\langle\mathrm{F}|\boldsymbol{\mu}| \mathrm{I}\rangle \mid \lesssim 10^{-2} \text {. }
$$

Though eq. (33) gives a small value when $\langle F|\mu| I\rangle$ is the non-zero, it is large enough to be significant when $\langle F|\mu| I\rangle$ vanishes in molecules with large atomic number elements.

We will neglect $\xi$ but retain $\mu^{\prime}$ in our general treatment. With these considerations we may give a final form to the integrated transition probability per second for the emission of a photon into $d \Omega_{k}$ with polarization $\lambda$. We have

$$
P_{\mathrm{IF}}^{\lambda}\left(\theta_{k}, \phi_{k}\right)=\left(\omega_{\mathrm{IF}}^{3} / h c^{3}\right)\left|\left[\langle\mathrm{F}|m| \mathrm{L}\rangle-k_{1} \times\left\langle\mathrm{F}\left|\boldsymbol{\mu}^{\prime}\right| \mathrm{I}\right\rangle-\mathrm{i}\left(\omega_{\mathrm{IF}} / 2 c\right) k_{1} \cdot\langle\mathrm{F}|q| \mathrm{I}\rangle\right] \cdot \boldsymbol{\Pi}_{k \lambda}\right|^{2},
$$

where $k=\omega_{\mathrm{IF}} / c, k_{1}=k / k$, and $P$ has been modified to make its dependence on $\theta_{k}$ and $\phi_{k}$, the angular coordinates of $k$, explicit. For future reference, we also define the total emission probability at $\theta_{k}, \phi_{k}$ for the I to $F$ transition as

$$
P_{\mathrm{IF}}\left(\theta_{k}, \phi_{k}\right) \equiv \sum_{\lambda} P_{\mathrm{IF}}^{\lambda}\left(\theta_{k}, \phi_{k}\right)
$$

and the total emission probability for the $I \rightarrow F$ transition as

$$
P_{\mathrm{IF}} \equiv \sum_{\lambda} \int P_{\mathrm{IF}}^{\lambda}\left(\theta_{k}, \phi_{k}\right) \sin \theta_{k} \mathrm{~d} \theta_{k} \mathrm{~d} \phi_{k}
$$

Eqs. (34), (35) and (36) are correct for a molecule of fixed orientation and are most easily applied when the molecular and laboratory coordinates are equivalent; this is the case for cubic symmetry molecules. Often, however, an oriented or high symmetry sample is not available and the emission results from a distribution of orientations with respect to our external coordinate system. We give a formal solution of this problem and refer the reader to others [3-5] for specific examples.

We define the laboratory coordinates by the orthonormal vectors $k_{1} \equiv 3, \pi_{1} \equiv 1$, and $\pi_{2} \equiv 2$. The molecular coordinates are designated by primes. We have, therefore, $l^{\prime}=\Sigma_{j} a_{i j} j$, where the $a_{i j}$ are functions of the Eulerian angles $\theta, \phi, \psi$, as defined by Goldstein [14]. The $a_{i j}$ are given by ref. [14] if the $\pi$ 's represent linear polarization vectors but not otherwise. The distribution of orientation before emission is given by $G(\theta, \phi, \psi)$, where

$$
1=\int_{0}^{2 \pi} \int_{0}^{2 \pi} \int^{\pi} G(\theta, \phi, \psi) \sin \theta \mathrm{d} \theta \mathrm{d} \phi \mathrm{d} \psi
$$

With these definitions, the probability of emission per second of a photon with polarization $\lambda$ and direction $k_{1}$ due to the I,F transition from those molecules in the sample with orientation $\theta, \phi, \psi$ with respect to the laboratory frame is 


$$
\begin{aligned}
& P_{\mathrm{IF}}^{\lambda k}(\theta, \phi, \psi)=\left[\omega_{\mathrm{IF}}(\theta, \phi, \psi) / h c^{3}\right] \mid\left(\mathrm{F}(\theta, \phi, \psi) \mid\left(\sum_{k} m_{k}^{\prime} a_{\lambda k}-\sum_{i, k} \mu_{k}^{\prime \prime} a_{i k}(3 \times i) \cdot \lambda\right.\right. \\
& -\left[\omega_{\mathrm{IF}}(\theta, \phi, \psi) /\left.2 c \sum_{i, j} q_{i j}^{\prime} a_{3 i} a_{\lambda j}\right|_{\left.|X(\theta, \phi, \psi)\rangle\right|^{2} G(\theta, \phi, \psi) .}\right.
\end{aligned}
$$

The average probability of emission from the entire sample in the direction $k_{1}$ with polarization $\lambda$ per unit time is

$$
P_{\mathrm{IF}}^{\lambda k}=\int_{0}^{2 \pi} \int_{0}^{2 \pi} \int_{\mathrm{IF}}^{\pi k}(\theta, \phi, \psi) \sin \theta \mathrm{d} \theta \mathrm{d} \phi \mathrm{d} \psi
$$

As discussed by previous authois [3-5], eq. (38) may differ markedly from (34) when the direction of the absorbing transition is at $90^{\circ}$ to the emitting transition, even for unpolarized incident light. For example, taking the above case with the absorbing transition along $z^{\prime}, G(\theta, \phi, \psi)=\left(3 / 16 \pi^{2}\right) \sin ^{2} \theta$. For $180^{\circ}$ excitation of an $x-y$ transition; $G=\left(3 / 32 \pi^{2}\right)\left(1+\cos ^{2} \theta\right)$.

In the absence of an external magnetic field, the transition energy and state functions are independent of orientation and eq. (37) is considerably simplified. Eq. (38) is completely applicable for any problem in which there is a time independent distribution of excited geometries; photoselection and stressed film spectra being two examples. The present theory does not incorporate the possibility of slow reorientation during emission $[4,5]$. For fast reorientation, as in solution, $G(\theta, \phi, \psi)=1 /\left(8 \pi^{2}\right)$.

\section{Special considerations in emission}

Before proceeding to a detailed discussion of the origin of linear and circularly polarized radiation, it is necessary to consider the emission process in a little more detail. There are two important aspects of emission that are foreign to the absorption process and there is a third consideration that is unusual.

1) Under constant incident energy and frequency excitation, the total number of excited molecules may change as a function of temperature. Further, of those molecules excited, the partitioning of energy among excited states may also be temperature dependent.

2) There are other processes besides emission of a photon (a phonon for example) that can lead to deactivation of the excited state(s).

3) There are usually several levels (possibly degenerate) from which emission takes place.

The problem is to design the polarized emission experiment in such a way as to avoid these difficulties. Given a few simple assumptions we can do so fairly well. With the additional assumption of Boltamann equilibrium among the levels, we can do very well.

Assumption 1. Each level involved in emission is depopulated by a group of mechanisms that lead to a first order decay rate with decay constant $\underline{k}_{I}$ for that state (I).

Assumption 2. At a fixed temperature, $T$, while the molecule is subjected to constant excitation, the integrated photon intensity of radiation emitted in direction $k_{1}$ with polarization $\lambda$ due to the transition $I \rightarrow F$ is given by

$$
\left[\underline{I}_{\mathrm{IF}}^{\lambda}\left(k_{1}\right)\right]^{0}=D(T) \underline{P}_{\mathrm{IF}}^{\lambda}\left(k_{1}\right) / \sum_{I}\left(k_{1} N_{1}\right)
$$

Given these two assumptions we now may simplify interpretation of experimental results. If the total integrated photon intensity due to the transition $I \rightarrow F$ measured in direction $k_{1}$ is $\left[I_{\mathbb{F}}\left(k_{1}\right)\right]^{0}$, then by eq. (39)

$$
\left[\underline{I}^{\lambda}\left(k_{1}\right)\right]^{0} /\left[\underline{I}_{1}\left(k_{1}\right)\right]^{0}=\sum_{\mathrm{I}, \mathrm{F}} \underline{P}_{\mathrm{I}, \mathrm{F}}^{\lambda} / \sum_{\mathrm{I}, \lambda, \mathrm{F}} \underline{P}_{\mathrm{IF}}^{\lambda}
$$

In eq. (40) we have removed the temperature dependence of everything but the $N_{1}$.

Assumption 3. The levels $I$ are in Boltzmann equilibrium. 


$$
N_{\mathrm{I}} / N=a_{\mathrm{I}} \exp \left(-W_{\mathrm{I}} / \kappa T\right) / \sum_{\mathrm{I}} d_{\mathrm{I}} \exp \left(-W_{\mathrm{I}} / \kappa T\right)
$$

With the aid of assumption 3 we may write eq. (40) as

$$
\left[I^{\lambda}\left(k_{1}\right)\right]^{0}=\sum_{1, F} P_{\mathrm{IF}}^{\lambda} \exp \left(-W_{\mathrm{IF}} / k T\right) d_{1} / \sum_{\mathrm{I}, \lambda, \mathrm{F}} P_{\mathrm{IF}}^{\lambda} \exp \left(-W_{\mathrm{IF}} / k T\right) d_{\mathrm{r}}
$$

Experimentally, if $I(\omega)$ is the photon intensity per unit frequency interval, we are interested in

$$
[\Delta]_{\alpha}^{n} \equiv \int\left[\underline{r}^{\lambda}(\omega)-\underline{I}^{\lambda^{\prime}}(\omega)\right]\left([\omega-a]^{n+3} / \omega^{3}\right) \mathrm{d} \omega
$$

These moments are generally useful and allow us to extract useful information from the emission that may be difficult or impossible to extract otherwise. For a general discussion of the method of moments as applied to MCD $[9,15]$, should be consulted. Also appendix VI of ref. [16] is of interest. The important point for now is that it is the ratio of two intensities, or two moments, that is simply related to our calculated transition probabilities.

\section{Relation to $C D, M C D$ and LPE}

In the previous sections a formula for the probability of emission as a function of polarization and direction of observation for a transition between two states 1 and $F$ of the molecule was derived. The notation of ref. [9] has purposely been used whenever possible so that we might compare our results in the emission case with those of Stephens in the absorption case. This will insure a unified terminology for both processes and as we shall show, our results for a particular geometry are simply related to his. We now proceed to give specific relations for the natural circularly polarized emission (CPE), the linearly polarized emission (LPE) and the MCE for the $180^{\circ} \mathrm{ex}$ perimental geometry.

The experimental geometry is chosen such that $k$ is co-linear with $H$. Further, we suppose that we have an oriented system ${ }^{\ddagger}$ so that the molecule fixed coordinates are identical with the laboratory fixed ones. We choose the $z$ axis to be directed from the sample, parallel with the external field $\boldsymbol{H}$, and toward the detector. Under these conditions

$$
k_{1}=\gamma_{2}, \quad \pi_{ \pm}=(i \pm i) / \sqrt{2}
$$

and we have

$$
P_{\mathrm{IF}}^{ \pm}(0,0)=\left(\omega_{\mathrm{IF}}^{3} / h c^{3}\right) \mid\left(\mathrm{F}\left|m_{ \pm}\right| \mathrm{D}\right) \pm \mathrm{i}\left(\mathrm{F}\left|\mu_{ \pm}^{\prime}\right| \mathrm{D} \pm\left(\omega_{\mathrm{IF}} / 2 c\right)\left\langle\mathrm{F}\left|q_{\mathrm{F}}\right| \mathrm{I}\right)^{2}\right.
$$

If the transition is reasonably dipole allowed, the terms quadratic in $\mu^{\prime}$ and $q$ may be safely neglected. With the assumption that we are dealing with a transition that is primarily electric dipole in nature, we have

$$
\left.P_{\mathrm{IF}}^{ \pm}(0,0)=\left(\omega_{\mathrm{IF}}^{3} / h c^{3}\right)\left\{\left.|\mathrm{F}| m_{ \pm}|\mathrm{D}|\right|^{2} \mp 2 \operatorname{Lm}\left[\left(\mathrm{F}\left|m_{ \pm}\right| \mathrm{I}\right)\left(\alpha\left|\mu_{\mathrm{F}}^{\prime}\right| \mathrm{F}\right)-\mathrm{i}\left(\omega_{\mathrm{IF}} / 2 c\right)\left(\mathrm{I}\left|q_{ \pm}\right| \mathrm{F}\right)\right)\right]\right\}
$$

Comparison of the quantity

$$
\underline{P}_{\mathrm{IF}}^{ \pm}=P_{\mathrm{IF}}^{ \pm}(0,0) N_{\mathrm{I}}
$$

with eq. (30) of ref. [9] gives

$$
\underline{P}_{\mathrm{IF}}^{ \pm}=\left[k_{ \pm}(\mathrm{F} \rightarrow \mathrm{I})\right]\left\{N_{\mathrm{I}} \omega_{\mathrm{IF}}^{3} / 4(\pi c)^{3}\left(N_{\mathrm{F}}-N_{\mathrm{I}}\right)\right\}
$$

F For systems of sufficient symmetry, 0 or $T_{d}$, for example, the relation of the space fixed to molecule fixed systern is arbitrary. Our equations here apply to solution's of molecules of this type. 
where $N_{\mathrm{I}}$ and $N_{\mathrm{F}}$ are the numbers of systems in the initial and final states, respectively. Since the CD and MCD equations derived in ref. [9] and elsewhere invariably assume $N_{\mathrm{I}}\left(N_{\mathrm{J}}\right.$ of ref. [9]) is zero, we may adopt all the CD and MCD equations with the substitution of $N_{\mathrm{I}}$ for $N_{\mathrm{F}}$ followed by the multiplication of the result by $\omega_{\mathrm{IF}}^{3} / 4 \pi^{3} c^{3}$. This procedure is perfectly accurate for the properties of a single pair of levels. When more than one level (which may be degenerate) is considered, the procedure of the previous section must be used.

In the absence of a field, the states $|I\rangle$ and $|F\rangle$ go into $|i \alpha\rangle,|f \beta\rangle$ where $\alpha$ and $\beta$ are enumeration indices for the possible degenerate levels $|i \alpha\rangle$, |f $\beta\rangle$. The differential photon emission probability between states $i$ and $f$ for natural circularly polarized light is given by

$$
\begin{aligned}
\Delta \underline{P}_{\mathrm{if}} & \equiv \sum_{\alpha, \beta}\left(\underline{P}_{\mathrm{i} \alpha, \mathrm{f} \beta}^{-}-\underline{P}_{\mathrm{i} \alpha, \mathrm{f} \beta}^{+}\right)=\left(2 \omega_{\mathrm{if}} N_{\mathrm{i}} / h c^{3}\right) \operatorname{Im}\left[\sum _ { \alpha , \beta } \langle \mathrm { f } \beta | m _ { x } | \mathrm { i } \alpha \rangle \left(\left\langle\mathrm{i} \alpha\left|\mu_{x}^{\prime}\right| \mathrm{f} \beta\right\rangle\right.\right. \\
& \left.\left.-\left(\omega_{\mathrm{if}} / 2 c\right)\left\langle\mathrm{i} \alpha\left|q_{z y}\right| \mathrm{f} \beta\right\rangle\right)+\sum_{\alpha, \beta}\left\langle\mathrm{f} \beta\left|m_{y}\right| \mathrm{i} \alpha\right\rangle\left(\left\langle\mathrm{i} \alpha\left|\mu_{y}^{\prime}\right| \mathrm{f} \beta\right\rangle+\left(\omega_{\mathrm{if}} / 2 c\right)\left(\mathrm{i} \alpha\left|q_{\mathrm{zx}}\right| \mathrm{f} \beta\right\rangle\right)\right] .
\end{aligned}
$$

With the assumption that we are dealing with a racemic mixture or a molecular system that is not optically active, eq. (44) becomes

$$
\underline{P}_{\mathrm{IF}}=\left(\omega_{\mathrm{IF}}^{3} / h c^{3}\right) N_{\mathrm{I}}\left|\left\langle\mathrm{F}\left|m_{ \pm}\right| \mathrm{I}\right\rangle\right|^{2}
$$

or

$$
\Delta P_{\mathrm{IF}}=\frac{N_{\mathrm{l}} \omega_{\mathrm{IF}}^{3}}{\left(N_{\mathrm{F}}-N_{\mathrm{I}}\right) 4 \pi^{3} c^{3}}[\Delta k(\mathrm{~F} \rightarrow \mathrm{I})]
$$

These are the appropriate equations for MCE. Note that the states $|\mathrm{F}\rangle$ and $|\mathrm{I}\rangle$ are now field dependent; $\Delta k(\mathrm{~F} \rightarrow \mathrm{I})$ is as defined in ref. [9].

In the case of linear polarization, one only needs to replace the circular basis vectors $\pi_{ \pm}$with the linear ones, $\pi_{1}$ and $\pi_{2}$. In fact, since $\pi_{1}^{*}=\pi_{1}$, the treatment is a little less complicated. From eq. (34) for example, for a molecular system oriented such that radiation is observed whose propagation vector is colinear with the molecular $z$ axis, the probability of $\pi_{1}$ polarization becomes

$$
P_{\mathrm{if}}^{\pi}(0,0)=\left(\omega_{\mathrm{IF}}^{3} / h c^{3}\right)\left|\left[\langle\mathrm{F}|m| \mathrm{D}\rangle-\gamma \times\left\langle\mathrm{F}\left|\mu^{\prime}\right| \mathrm{D}\right\rangle-\mathrm{i}\left(\omega_{\mathrm{IF}} / 2 c\right) \gamma \times\langle\underset{\mathrm{F} \mid q}{q} \mid \mathrm{D}\rangle\right] \cdot \pi_{1}\right|^{2}
$$

The analysis of MLD (sometimes called the Voigt effect) is slightly more complicated than that of MCD because it is a second order effect. That is, the signal, $\Delta I=I_{-x}-I_{-y}$, in the presence of a field depends on $H^{2}$ rather than $H$. MLD $[17,18]$ contains potentially different information than MCD, but it has not experienced anything like the growth rate of MCD, perhaps because there are no commercial MLD machines available. Since our interest here is with zero and first order field polarization, we will not develop the parameters for magnetically induced linear polarization of emission. It should be noted, however, that any attempt to do so should take second order contributions to the energy due to the magnetic field into account.

\section{MCE parameters}

In the standard development of $\mathrm{MCD}$, it is customary to express the first order magnetic field dependence of the analog of eq. (47) in terms of three parameters. These parameters are arrived at by considering the field dependence of the populations $N_{\mathrm{I}}$ and the state functions $|\mathrm{F}\rangle$ and $|\mathrm{I}\rangle$. Roughly speaking, the first order dependence on $\boldsymbol{H}$ of $N_{1}$ leads to a parameter, $C$, which is proportional to $(k T)^{-1}$ if degeneracies, in the absence of $\boldsymbol{H}$, are present in the initial state(s). First order shifts in energy when either initial or final states are degenerate, are contained in the parameter, $A$. Because the character of the initial and final states will always depend on $\boldsymbol{H}$ via "second order interactions", there is finally the parameter $B$. The $C$ and $B$ terms generally have the same frequency depen- 

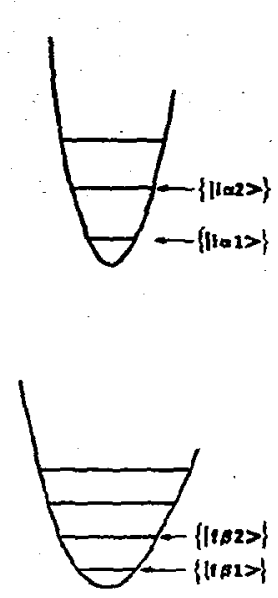

$\mathbf{H}=\mathbf{O}$
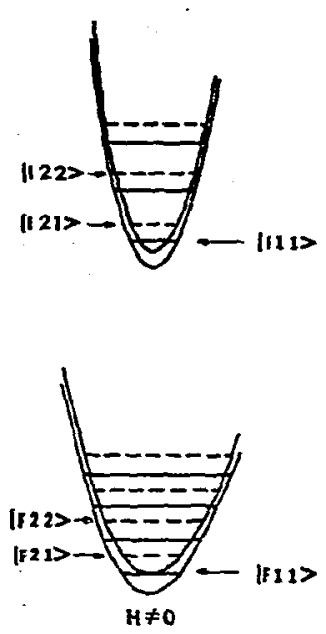

Fig. 1. Schematic diagram of a few molecular states in the context of the Born-Oppenheimer approximation. Magnetic field off and on.

dence as the absorption (except for a signed constant) while the $A$ terms are somewhat like the differential of the absorption distribution.

In this section, within the Rigid Shift Approximation (vide infra), we will present the equivalent parameterization for the MCE case. We begin by expanding the notation of eqs. (11) and (12). Fig. 1 is an exaggerated conceptual view, in the context of the Born-Oppenheimer approximation, of the eigenstates of $\underline{H}^{0}$ and $\underline{H}^{\mathrm{M}}$. With this picture in mind we have

$$
\underline{H}^{\mathrm{M}}|\mathrm{I} \epsilon, \mathrm{r}\rangle=W_{\mathrm{IeI}}|\mathrm{I} \epsilon, \mathrm{r}\rangle, \quad \underline{H}^{0}|\mathrm{i} \epsilon, \mathrm{r}\rangle=W_{\mathrm{ir}}|\mathrm{i} \epsilon, \mathrm{r}\rangle,
$$

where

$$
|i \epsilon, \mathrm{r}\rangle=\lim _{H \rightarrow 0}|I \epsilon, \mathrm{r}\rangle .
$$

We should also note that there will generally be more level sets than $\{|i \epsilon, r\rangle\}$ and $\{|f \phi, r\rangle\}$; these other levels are omitted from the diagram for the sake of clarity. Because the energy shift produced by the field is seldom more than a few wavenumbers, perturbation theory is usually sufficient for determining the $|I \epsilon, r\rangle$ from the $\{|j \delta, r\rangle\}$, where $|j \delta, r\rangle$ is a generic zero field eigenstate. In fact the first order theory is generally sufficient, in which case

$$
|\mathrm{J} \delta, \mathrm{r}\rangle=|\mathrm{j} \delta, \mathrm{r}\rangle+\sum_{\substack{\mathbf{k}, \phi, \mathrm{r} \\ \neq(\mathrm{j}, \delta, \mathrm{r})}} \frac{\left\langle k \phi, \mathrm{r}\left|\boldsymbol{\mu}^{\prime}(\mathrm{j} \delta, \mathrm{r}\rangle \cdot \boldsymbol{H}\right| \mathrm{k} \phi, \mathrm{r}\right\rangle}{\hbar \omega_{\mathrm{kj}}} .
$$

In general, the shape of the zero field emission is not that predicted by the quantum theory for a single transition. Some reasons for this in condensed media are:

1) There is a continuum of phonon states to which the transition may occur.

2) Inhomogeneities in the matrix cause the local environment, and therefore $\underline{H}^{0}$, to be a function of position in the matrix.

3) The transition from vibronic state $|i \epsilon, \tau\rangle$ to $|f \phi, s\rangle$ may be nearly degenerate with the one from $\left|i^{\prime} \epsilon^{\prime}, r^{\prime}\right\rangle$ to $\left|f^{\prime} \phi^{\prime}, s^{\prime}\right\rangle$. 
These effects, taken together, can lend to emission bands which are $10^{3} \mathrm{~cm}^{-1}$ wide. Clearly, the detailed calculation of location and intensity of every transition involved is impractical and, for now, impossible. One method for circumventing these difficulties is to assume a shape function, $\omega^{3} f(\omega)$ such that

$$
\int f(\omega) \mathrm{d} \omega=1
$$

This approach is quite reasonable for line spectra where the width is due strictly to the phonon continuum and to small inhomogeneities in the matrix $\left(<150 \mathrm{~cm}^{-1}\right.$ wide in the visible). In this case we may accurately write +

$$
\underline{I}=\sum_{\mathrm{i}, \mathrm{f}} \omega^{3} \underline{T}_{\mathrm{if}} f_{\mathrm{if}} / \sum_{\mathrm{i}}\left(k_{\mathrm{i}} N_{\mathrm{i}}\right)
$$

When bands ( $>200 \mathrm{~cm}^{-1}$ wide) are considered, this is a dangerous procedure. Buried beneath a band may be several principal transitions followed by long progressions in one, or more, vibrational mode. In the case where all the progressions are due to a totally symmetric vibration, the transition probability for each component of the progression is related to the transition probability of the progression origin $[2 a, 19]$. In this case, a modified form of eq. (53) may be used

$$
\underline{I}_{\mathrm{if}}=D(T) T_{\mathrm{i} 0, \mathrm{f} 0} \omega^{3} f_{\mathrm{if}}(\omega) / \sum_{\mathrm{i}} \underline{k}_{\mathrm{i}} N_{\mathrm{i}}
$$

provided that (a) the Bom-Oppenheimer approximation is valid, (b) the progression involves a totally symmetric mode, and (c) the shape of each vibrational component is the same.

With these restrictions in mind we may give the form of $\underline{I}$ for several progression origins as

$$
\underline{I}=D(T)\left[\sum_{\mathrm{i} \rightarrow \mathrm{f}} \underline{T}_{\mathrm{if}} \omega^{3} f_{\mathrm{if}}\right] / \sum_{\mathrm{i}} \underline{k}_{\mathrm{i}} N_{\mathrm{i}},
$$

where $T_{\text {if }}$ is evaluated for electronic states $i$ and $f$ only. We will adopt eq. (54) for the rest of this subsection since it is equally valid for line spectra.

When a field is applied, the shape function is affected. In the so-called rigid shift approximation $[8,9,15]$, the potential surfaces (F1 and F2 for example) in fig. 1 move rigidly away from each other, and if no new transitions are induced, the shape of the new $I \epsilon \rightarrow F \phi$ transition is just that for $i \rightarrow f$ shifted in energy.

$$
f_{\mathrm{I} \in, \mathrm{F}, \phi}(\omega)=f_{\mathrm{if}}\left[\omega-\left(\omega_{\mathrm{I} E, \mathrm{~F} \phi}-\omega_{\mathrm{if}}\right)\right] \text {. }
$$

When the Zeeman splitting is much smaller than the line width we have

$$
f_{\mathrm{IE}, \mathrm{F} \phi}=f_{\mathrm{if}}-2 \pi f_{\mathrm{if}}^{\prime}\left(w_{\mathrm{I} \varepsilon}-W_{\mathrm{i}}-W_{\mathrm{F} \phi}+W_{\mathrm{f}}\right) / \hbar \text {. }
$$

At this point we have considered all the necessary components for the parameterization of first order MCE. We will restate the assumptions and then, utilizing direct substitutions, write down the results.

1) Eq. (52) is valid.

2) Eq. (54) is valid.

3) Eq. (55) is valid.

4) The molecular system shows no natural optical activity or is composed of a racemic mixture.

5) The transition is allowed by an electric dipole mechanism.

6) The geometry of the experiment is "head-on".

7) The unpolarized spectrum in zero field is identical to the unpolarized spectrum with the field on (to first order in $H$ ).

\footnotetext{
\# Omitting the $\mathrm{r}, \mathrm{s}$ indices for the sake of notational simplicity, $T_{\mathrm{if}}$ is measured in some direction $k_{1}$, if $k_{1}=\gamma, \underline{T}_{\mathrm{if}}^{ \pm}(0,0)=\underline{T}_{\mathrm{if}}^{ \pm}=$ $\left(\mid K \cap m_{ \pm}\left[\mathrm{i}||^{2} / h c^{3}\right) N=P_{\mathrm{if}}^{ \pm}(0,0) / \omega^{3}\right.$.
} 
8) Assumptions 1,2 and 3 (only for levels degenerate in zero field) of section 3 obtain with $W_{1}$ given by the first order perturbation theory.

9) The system is composed of molecules of cubic symmetry. Altemately, they may be of any symmetry provided that they are oriented with respect to the laboratory frame or are experiencing fast rotational relaxation. In the last case, the form of the parameters given by Stephens for solutions is appropriate. For $\mathrm{C}_{3}$ symmetry, the solution parameters may be used for rigid randomly oriented samples with $\approx 40 \%$ error.

$$
l=\sigma(T)\left[\sum_{\mathrm{i} \rightarrow \mathrm{f}} \omega^{3} D^{\prime}(\mathrm{f} \rightarrow \mathrm{i}) f_{\mathrm{if}} N_{\mathrm{i}}\right]
$$

where

$$
\begin{aligned}
& \sigma(T)=n^{3} \alpha^{2} D(T) /\left[3 k c^{3} \sum_{\mathrm{i}} \underline{k}_{\mathrm{i}} N_{\mathrm{i}}\right] . \\
& \Delta I=-\sigma(T) \sum_{\mathrm{i} \rightarrow \mathrm{f}} N_{\mathrm{i}} \omega^{3}\left\{A^{\prime}(\mathrm{f} \rightarrow \mathrm{i}) f_{\mathrm{if}}^{\prime} / \hbar+\left[B^{\prime}(\mathrm{f} \rightarrow \mathrm{i})+C^{\prime}(\mathrm{f} \rightarrow \mathrm{i}) / k T\right] f_{\mathrm{if}}\right\} H,
\end{aligned}
$$

$A^{\prime}(\mathrm{f} \rightarrow \mathrm{i}), B^{\prime}(\mathrm{f} \rightarrow \mathrm{i})$, and $D^{\prime}(\mathrm{f} \rightarrow \mathrm{i})$ are exactly those parameters given in refs. [8,9], except that $d_{\mathrm{i}}$ replaces $d_{\mathrm{f}} ;$ note also that the transition operators are unnormalized. $C^{\prime}(f \rightarrow i)$ is modified as in eq. (59) because of the population contribution.

$$
C^{\prime}(\mathrm{f} \rightarrow \mathrm{i})=-\frac{3}{4 d_{i}} \sum_{\epsilon \rightarrow \phi}\left|\left\langle f \phi\left|m_{-}^{\mathrm{s}}\right| \mathrm{i} \epsilon\right\rangle\right|^{2}-\left|\left\langle f \phi\left|m_{+}^{\mathrm{s}}\right| \mathrm{i} \epsilon\right\rangle\right|^{2}\left\langle\mathrm{i} \epsilon\left|\mu_{z}^{\prime}\right| \mathrm{i} \epsilon\right\rangle
$$

We have successfully parameterized the MCE equations, for the case where assignment of a shape function is appropriate, in a form similar to that used for MCD. The conditions under which they are applicable have been stated. The relative sizes of the $A^{\prime}, B^{\prime}$ and $C^{\prime}$ have been discussed elsewhere. [8,9] and we note here that if only the excited state is degenerate then $A^{\prime}=C^{\prime}$ (as opposed to the case in MCD where if only the ground state is degenerate then $A=-C$ ).

\section{Acknowledgement}

I wish to thank Professor G.A. Crosby for his support and encouragement. Financial support was provided by AFOSR(NC)-OAR USAF grant AFOSR-76-2932 and by the National Science Foundation in the form of a postdoctoral fellowship.

\section{References}

[1] B. Ehrenberg and I.Z. Steinberg, J. Amer. Chem. Soc. 98 (1976) 1293;

A. Gafni and 1.z. Steinberg, Photochem. and Photobio. 15 (1972) 93;

H. Sixl and M. Schwoerer, Chem. Phys. Letters 6 (1970) 21;

Emeis and Oosterhoff, Chem. Phys. Letters 1 (1967) 129.

[2] M. Fontana and J. Davis, Phys. Rev. Lett. 23 (1969) 974;

Marrone and Kabler, Phys. Rev. Lett. 27 (1971) 1283;

Shatwell and MoCaffery, Mol. Phys. 30 (1975) 1489;

C. Luk et al., Chem. Phys. Letters 34 (1975) 147;

F.S. Richardson et al., J. Phys. Chem. 80 (1976) 2228.

[2a] K.W. Hipps, Ph.D. Thesis, Washington State University (1976).

[3] J. Snir and J. Schellman, J. Phys. Chem. 78 (1974) 387.

[4] I.Z. Steinberg and B. Ehrenberg, J. Chem. Phys. 61 (1974) 3382. 
[5] J.P. Riehl and F.S. Richardson, J. Chem. Phys. 65 (1976) 1011.

[6] E.A. Powers and T. Thirunamchandran, J. Chem. Phys. 60 (1974) 3695.

[7] H. Eyring and D. Caldwell, Theory of optical activity (Wiley-Interscience, New York, 1971).

[8] P.J. Stephens, Ann. Rev. Phys. Chem. 25 (1974) 201.

[9] P.J. Stephens, J. Chem. Phys. S2 (1970) 3489.

[10] J.J. Sakurai, Advanced quantum mechanics (Addison-Wesley, Reading, 1967).

[11] B. DiBartolo, Optical interactions in solids (John Wiley and Sons, New York, 1968).

[12] W. Heitler, The quantum theory of radiation (Oxford University Press, Oxford, England, 1967).

[13] J.S. Grifrith, The theory of transition-metal ions (Cambridge University Press, London, 1971).

[14] H. Goldstein, Classical mechanics (Addison-Wesley, Reading, Mass., 1965).

[15] C.H. Henry, S.E. Schnatterly and C.P. Slickter, Phys. Rev. 137A (1965) 583.

[16] K. Rebane, Impurity spectra of solids (Plenum, New York, 1970).

[17] A.C. Boccara, J. Ferre, B. Briat, M. Billardson and J.P. Badoz, J. Chem. Phys. 50 (1969) 2716.

[18] J. Ferre, B. Briat, C. Paparoditis, S. Pokrzywnicki and R. Siryanarayane, Solid State Commun. 11 (1972) 1173.

[19] K.W. Hipps, G.A. Merrell and G.A. Crosby, J. Phys. Chem. 80 (1976) 2232. 